\title{
In-Line Measurement of Color and Total Phenolics during Red Wine Fermentations Using a Light-Emitting Diode Sensor
}

\author{
Nicholas L. Shrake, ${ }^{1}$ Rajeevan Amirtharajah, ${ }^{1}$ Charles Brenneman, ${ }^{2}$ \\ Roger Boulton, ${ }^{2 *}$ and André Knoesen ${ }^{1}$
}

\begin{abstract}
An in-line color and total phenolic sensor is described to track the color and total phenol evolution during red wine fermentations. The sensor uses multiple light-emitting diodes (LEDs) spanning the ultraviolet and visible spectrum, in particular 280 and $525 \mathrm{~nm}$. The performance of phenolic sensor was evaluated by analyzing fermentation samples collected from multiple red wine fermentations performed during the 2012 season. The study confirmed that the LED phenolic sensor measurements strongly correlate with measurements performed with a reference UV-vis spectrophotometer and that in-line measurements can be made in a practical manner after removal of yeast and pulp with a $2.0 \mu \mathrm{m}$ filter. The use of a $100 \mu \mathrm{m}$ path-length flow cell avoids the need for dilution, making in-line measurements possible. The sensor provides in-line measurement on the evolution of the total phenolic content and color extraction patterns during red wine fermentations.
\end{abstract}

Key words: in-line, real-time, total phenolics, red wine color, fermentation, extraction pattern

In red wine fermentations, color and phenolic compounds are extracted from the grape skins and additional phenolic components are extracted from the seeds into the must. The pigments and phenolic compounds extracted during contact determine the color and mouthfeel of the wine (Thorngate and Noble 1995, Brossaud et al. 2001). In sensory terms, phenolic compounds are closely associated with astringency and sometimes bitterness, and it is of interest to measure these compounds in real-time and to follow the extraction pattern in all stages of contacting and fermentation. Currently, the measurement of phenolics during fermentation involves manually sampling on a regular schedule and storing in a refrigerator, and at a later stage completing the analysis. This approach is slow and the information is delayed and less useful for making skin contact decisions in an ongoing fermentation. An in-line sensor that monitors phenolic information in real-time is the preferable alternative.

Winemakers make phenolic measurements based on either chromatography or spectroscopy. High performance liquid chromatography (HPLC) allows precise measurement of individual phenolic compounds (Donovan et al. 1998, Peng et al. 2002). While accurate, HPLC systems are technical-

\footnotetext{
${ }^{1}$ Department of Electrical and Computer Engineering and ${ }^{2}$ Department of Viticulture and Enology, University of California, Davis, CA 95616.

*Corresponding author (rbboulton@ucdavis.edu)

Acknowledgments: Nicholas L. Shrake is supported by a T.J. Rodgers Fellowship in electrical and computer engineering. The authors gratefully acknowledge the assistance of Babak Taheri and Mark Holst during the project and the donation of the Pinot noir grapes from T.J. Rodgers.

Manuscript submitted Feb 2014, revised Jul 2014, accepted Aug 2014

Copyright (C) 2014 by the American Society for Enology and Viticulture. All rights reserved.

doi: 10.5344/ajev.2014.14023
}

ly not feasible for in-line setups due to long analysis times (70+ minutes) and significant instrument cost. Spectroscopy measurements can be used to quantify tannin, anthocyanin, and other non-tannin phenols. Color information from the anthocyanins is contained in an absorption band in the 495 to $545 \mathrm{~nm}$ region. The total phenol measurements are made around $280 \mathrm{~nm}$, which corresponds to an intense absorption band associated with the benzene ring common to all phenolic compounds. In the simplest optical absorbance analysis, fermentation samples and red wines are analyzed for color at $525 \mathrm{~nm}$ and total phenolics at $280 \mathrm{~nm}$ (Somers 1998, Harbertson et al. 2006). More specific phenolic compounds can be quantified with chemical assays or multivariate chemometric models for those assays. Chemical assays obtain information by a reaction between the sample and a reagent to produce high absorbance at an indicator wavelength (Harbertson et al. 2003). The use of chemical assays is avoided here, however, because the procedure is destructive and not adaptable to an in-line approach due to the multistep procedure involving long incubation and centrifugation times. Multivariate chemometrics based on near infrared and UV-vis spectra have been explored (Cozzolino et al. 2004, Skogerson et al. 2007) as a tool for rapid and real-time phenolic estimation of subgroups of the total phenols. Of particular importance in red wine fermentation are measurements in the ultraviolet region (250 to $320 \mathrm{~nm}$ ) where the absorbance is very large (up to $75 \mathrm{AU}$ in a $1 \mathrm{~cm}$ path length). The practice of dilution (Skogerson et al. 2007) to bring the UV absorbance on-scale undermines intact color estimation due to the disruption of copigmentation effects (Boulton 2001). Optical measurements can be made on undiluted samples only if a short optical path length (less than $1 \mathrm{~mm}$ ) is used.

An in-line optical spectrophotometer with a small pathlength cell, 100 to $500 \mu \mathrm{m}$, should be able to follow the 
evolution of color and total phenolics during a red wine fermentation, using undiluted samples. In practice, in-line optical measurements are challenging because of light scattering caused by bubbles and suspended particles. While a commercial spectrophotometer could be adapted to perform in-line measurements, it is not a desired option because of high cost, power, and space involved. A conventional white light source is limited because of low photon flux at most wavelengths, which necessitates expensive photodetectors such as photomultiplier tubes or avalanche-based photodiodes. In contrast, solid-state light-emitting diodes (LEDs) offer a low-cost option with a large photon flux at the chosen wavelength that allows the use of silicon photodiodes. Custom LED-based photometers have been investigated previously for transmission and reflectance measurement applications in analytical chemistry (Dasgupta et al. 2003). Several groups have adopted LED colorimeters for field trials in processing monitoring (Hauser et al. 1995) and wastewater monitoring (Beaton et al. 2011) because of their low cost, small form factor, and minimal power consumption. The wide absorbance band of the total phenolic and color compounds in red wine made using an LED as an optical source an attractive option.

The aim of this study was to develop and evaluate an inline sensor capable of following the extraction of color and total phenols during red wine fermentations.

\section{Materials and Methods}

In-line color and total phenol sensor. The multispectral LED colorimeter is shown in Figure 1. The main factor that determined colorimeter design was the requirement of making absorption measurements in the UV $(280 \mathrm{~nm}$ and 320 $\mathrm{nm})$ in a quartz transmission flow cell with a short $100 \mu \mathrm{m}$ path length and a window size of $8 \mathrm{~mm} \times 40 \mathrm{~mm}$ (584.4-Q0.1; Starna Cells, Atascadero, CA). A transmission optical measurement was selected since spectroscopic absorption measurements are performed over a well-defined optical path length. The UV $280 \mathrm{~nm}$ LED (UVTOP-280TO18BL; SETI Inc., Columbia, SC) and UV $320 \mathrm{~nm}$ LED (UVTOP325TO18BL; SETI Inc.) are mounted directly to the flow cell to minimize extraneous absorption of UV photons. Both
UV LEDs include a quartz ball lens (spot size of $1.5 \mathrm{~mm}$ at a focal point of $2 \mathrm{~cm}$ ) that is used to improve collection efficiency for UV photons onto the $2.54 \mathrm{~mm}$ diameter UV photodiodes (model PC10-2-TO5; Pacific Silicon, Westlake Village, CA). Measurements were also made with six visible wavelength LEDs, $420 \mathrm{~nm}$ (OUE8A420Y1; Optek Technology Inc., Carrollton, TX), $470 \mathrm{~nm}$ (WP7113QBC/D; Kingbright, Taipei, Taiwan), $505 \mathrm{~nm}$ (OVLGC0C6B9; Optek Technology Inc.), $525 \mathrm{~nm}$ (C503B-GAS-CB0F0792; Cree Inc., Durham, NC), $565 \mathrm{~nm}$ (TLHG4900; Vishay, Malvern, PA), and 630 $\mathrm{nm}$ (WP813SRC/F; Kingbright). While visible measurements could be made with a longer path-length cell, for simplicity of design, it was decided to use the same path length for all measurements. The visible LEDs were coupled into the cell by multimode fluorinated polymer optical fibers (EKSA model 02-536; Edmund Optics, Barrington, NJ). The use of polymer fiber to deliver light into the cell was required because of space requirements imposed by the optical flow cell. The visible LED sources were coupled into optical fibers by end coupling. The efficiency of coupling was maximized by grinding the epoxy casing of LEDs to less than $0.5 \mathrm{~mm}$ distance from the semiconductor die surface and then optical polishing of the surfaces $(1 \mu \mathrm{m}$ grit, model LFG1P; Thor Labs, Newton, NJ). The polished LEDs were mounted on a printed circuit board. The multimode polymer optical fibers were aligned to the LEDs by $15 \mathrm{~mm}$ long tubes made into aligning fixtures into which the fibers are permanently affixed by hot glue (model 2013548; Ace Hardware Brand., Oak Brook, IL). The visible wavelength photodiodes (model SFH2701; OSRAM Opto Semiconductor Inc., Munich, Germany) were selected for their small area $(1.65 \mathrm{~mm} \times 3.35 \mathrm{~mm})$ and are required, due to the small window area of the optical flow cell.

The distance from the UV LED or optical fiber to the photodiode is fixed at $2.6 \mathrm{~mm}$. The numerical aperture for the ball-lens UV LED is 0.05 (3-degree half angle). The numerical aperture for the polymer optical fiber is 0.51 (30-degree half angle). The numerical aperture for the blue-enhanced UV photodiode is 0.53 (32-degree half angle). The numerical aperture of visible-wavelength photodiode is 0.87 (60-degree half angle). The focused beam of the UV LED source fills

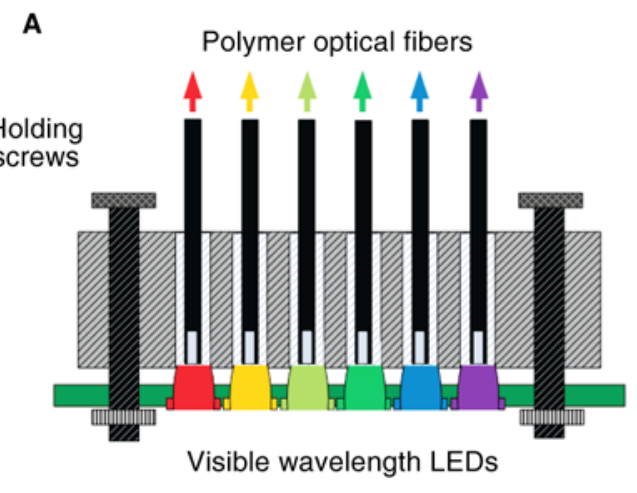

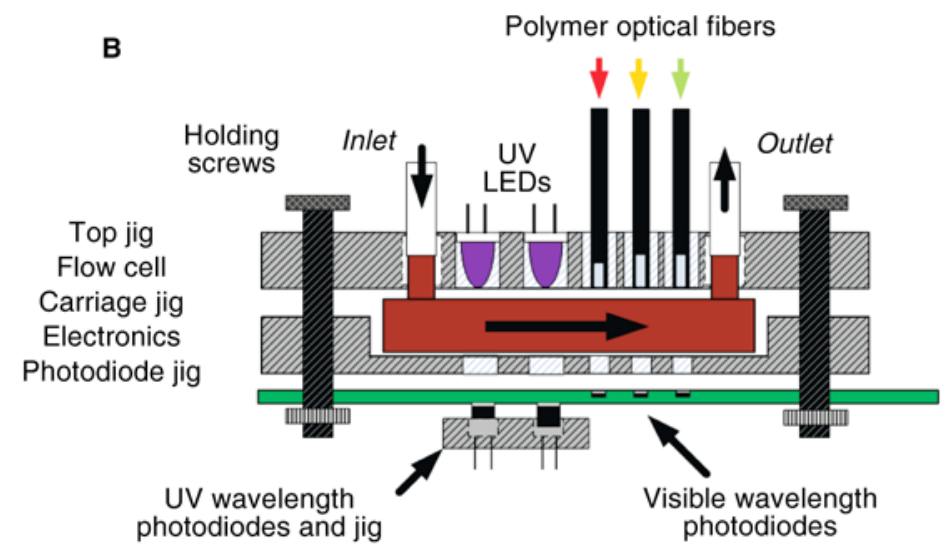

Figure 1 Cross section of the multiwavelength LED sensor for phenolic and color analysis. (A) Optical fibers are polished and coupled to visible wavelength LED sources on a circuit board using a jig. (B) Samples are pumped into the inlet of the flow cell. Two UV LEDs are mounted on the cell and six visible wavelengths are coupled via polymer optical fibers. 
the UV photodiode surface, resulting in maximum collection efficiency. The fiber, owing to its large numerical aperture, is a dispersive beam and much of the light falls outside the detectors' small area. However, the power coupled into the fiber is sufficiently high that loss of light is not of concern.

A transimpedance (OPA124; Texas Instruments, Dallas, TX) electronic front-end was used for all visible wavelengths, and a switched integrator (ACF2101; Texas Instruments) was used for the UV wavelengths 280 and $320 \mathrm{~nm}$. The switched integrator timing signals for hold, select, and reset were generated by a microcontroller (PSoC CY8C3866AXI-040; Cypress Semiconductor, San Jose, CA). A bipolar 18-volt power supply (model E3620A; Agilent, Santa Clara, CA) is used with two linear regulators to create 15 Volt and -15 Volt power supplies (MC79M15CDTRKG, MC78M15CDTRKG; ON Semiconductor, Phoenix, AZ). The 15 Volt and -15 Volt supplies are used to power the six transimpedance amplifiers and single switched integrator. The amplifier provides sufficient gain such that the maximum photocurrent will output the full scale system voltage. The maximum photocurrent is around $40 \mu \mathrm{A}$ for visible LEDs and $2 \mu \mathrm{A}$ for the UV LEDs. The gain of all transimpedance channels is set to to 400,000 (V/I) to produce a full scale output of -15 Volts for the $40 \mu \mathrm{A}$ photocurrent. The integration time is set to 500 microsecond for an effective gain of 5,000,000 (V/I) to produce a full scale output voltage of 10 volts for the $2 \mu \mathrm{A}$ photocurrent. The output voltage is level-shifted to a 5 Volt full scale before being digitized. The 8 LEDs are sequentially pulsed in a 1 sec interval. The transimpedance and integrator outputs are multiplexed and digitized by a 12-bit successive approximation analog to digital converter onboard the microcontroller. The instrument is controlled and data collected over a USB interface by a graphical user interface (LabView, National Instruments, Austin, TX). The LED colorimeter makes 64 absorbance measurements at each wavelength and reports the mean and standard deviation of the analysis.

Red Wine Fermentations. During the 2012 harvest, 256 samples were collected at various stages during red wine fermentations performed at the UC Davis Department of Viticulture and Enology Pilot Winery. Grapes of the cultivars Vitis vinifera var. Syrah, Cabernet Sauvignon, and Barbera fruit were picked from the UC Davis Vineyard and the Cabernet franc and Malbec was from UC Oakville Vineyard. The fruit was destemmed and crushed (Bucher Vaslin, Charlonne-surLoire, France) and placed directly into $200 \mathrm{~L}$ stainless-steel research fermentors (Sharpsville Container Corp., Sharpsville, PA). The fermentations were controlled for temperature and mixing was monitored by an integrated fermentation control system developed by Cypress Semiconductor. These units were programmed to control a chosen fermentation temperature and were set to automatically pump over the fermentation every three hr. In all, 256 samples were taken from thirteen individual fermentations that included Syrah (156), Cabernet Sauvignon (66), Cabernet franc (12), Barbera (13), and Malbec (9).

In-line Study Sample Collection. The evolution of total phenolics and color were followed during the fermentation of two Cabernet fruit and one Pinot noir fermentations by making in-line measurements with the LED sensor. The inline measurement method was validated on two Cabernet Sauvignon fermentations. The block diagram for the fluidic system is shown in Figure 2. Synchronized with fermentation pump-overs, the in-line system automatically sampled every five hr over the fermentation period of $140 \mathrm{hr}$. A Y-fluid coupler was used to alternate pushing water or wine through the optical cell. A peristaltic pump (model 14-375-40; ColeParmer, Chicago, IL) pulled a wine sample, and pushed the sample through a filter $(2.0 \mu \mathrm{m}$ pore size membrane filter, model AP2504700; Millipore, Billerica, MA, and 47mm inline polycarbonate filter holder, model 1119; Pall Gelman, Port Washington, NY) and into the flow cell. An identical peristaltic pump pushed deionized water through the flow cell. The in-line sampling protocol was as follows: $1.5 \mathrm{~min}$ of flushing the optical cell with water, 1.5 min with water "reference" measurement, $1.5 \mathrm{~min}$ of wine flowing through the cell, and $1.5 \mathrm{~min}$ of sample measurement. A total of $100 \mathrm{~mL}$ of wine is shunted during the 3 -min pump time. The water and wine flow is halted during the absorbance measurements to avoid bubble contamination. The peristaltic pumps were controlled using LabView (National Instruments) via a solid-state AC relay (Power Switch Tail 2; Adafruit, New York, NY) and a digital interface (model 6009; National Instruments). Control samples were also manually drawn coinciding with the automatic sample times, placed in $50 \mathrm{~mL}$ plastic tubes (model 14-375-150; Fisher Brand, Waltham MA) and immediately stored in a refrigerator at $-4^{\circ} \mathrm{C}$ for later analysis.

Manual Study Sample Collection. Manual samples were collected from 11 fermentations every $12 \mathrm{hr}$, placed in $50 \mathrm{~mL}$ centrifugation tubes (model 14-375-150; Fisher Brand) and immediately stored in a refrigerator at $-4^{\circ} \mathrm{C}$. A total of 256 samples were collected and analyzed.

Laboratory Sample Analysis. $14 \mathrm{~mL}$ samples were extracted and transferred to $15 \mathrm{~mL}$ plastic centrifuge tubes

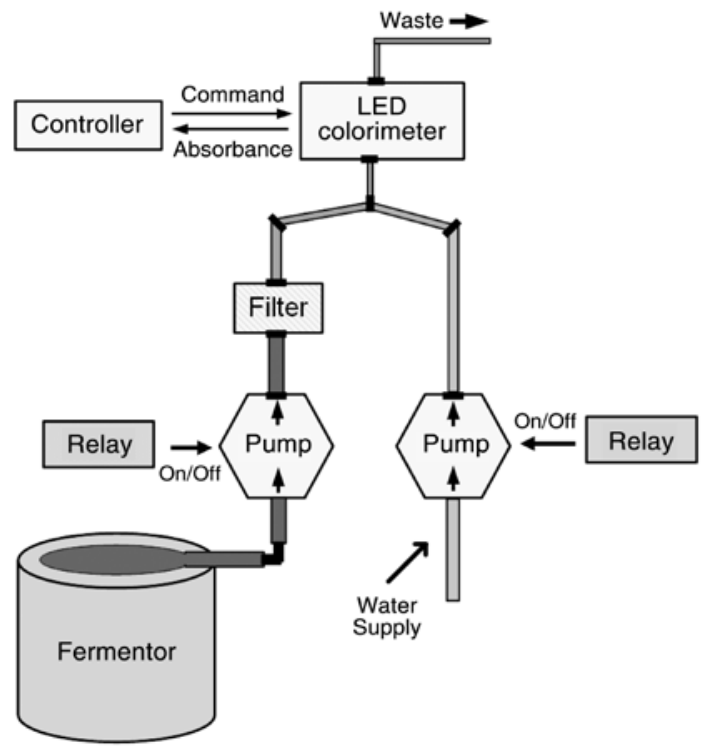

Figure 2 Block diagram of in-line sampling configuration during red wine fermentation. A PC controller applies an On/Off signal to a relay block to alternate peristaltic pumps. 
(model 339650; ThermoFisher, Waltham, MA). The extraction and transfer procedure was repeated for the entire manual sample bank $(\mathrm{n}=256)$. The samples were centrifuged (model 225; Fisher Scientific, Waltham, MA) for $10 \mathrm{~min}$ and left on a bench for $30 \mathrm{~min}$ to reach room temperature. UV-visible spectra were collected with a spectrophotometer (model HP 8453; Agilent) using a $100 \mu \mathrm{m}$ path-length cuvette (584.4-Q-0.1; Starna Cells). Readings were also collected using the LED sensor. The UV-vis and LED sensor measurements were run on single samples (no replicates). Deionized water was used for the reference scan. For both instruments, samples were pumped into the cell using a sipper peristaltic pump (model 89052B; Agilent) with a $30 \mathrm{sec}$ deposit and $10 \mathrm{sec}$ wait time before analysis.

\section{Results}

The performance of the LED colorimeter was validated using an in-line and laboratory experimental configuration. The in-line study examines the effect of interference from residual particles in the wine solution after a $2.0 \mu \mathrm{m}$ filtration. Samples were manually drawn at the in-line sampling time and centrifuged offline before being analyzed using the LED colorimeter. The study quantifies the absorbance offset contribution from particles by comparing measurements made on red wine fermentation samples using either the $2.0 \mu \mathrm{m}$ filter or centrifuge. A second investigation was used to determine if the LED colorimeter could be used as a replacement for a UV-vis spectrophotometer. A large number of fermentation samples were collected and measured using the LED colorimeter and UV-vis after a centrifuge step. A strong correlation was found between absorbance measurements made with the LED sensor and UV-vis spectrophotometer for total phenolic $\left(\mathrm{R}^{2}=0.98\right)$ and color $\left(\mathrm{R}^{2}=0.95\right)$.
A phenolic extraction plot comparing in-line and laboratory absorbance measurements during fermentation is shown in Figure 3. The phenolic measurements shown are made with the LED colorimeter instrument. The in-line method used a pump to push fermentation samples through a filter after which total phenols and red color were measured and recorded. The laboratory method used a centrifuge on samples that were manually collected at the in-line sampling times. The absorbance measurements for the in-line method were always greater than the laboratory centrifuge system. The absorbance offset is most pronounced for the total phenolic $(280 \mathrm{~nm})$ measurement with the background offset of the in-line method approaching $50 \%$ of the total absorbance measured. In contrast, the color $(525 \mathrm{~nm})$ measurement had a background offset of at most $25 \%$ of the total absorbance. The background offset arises from suspended particles (yeast and pulp) in the sample. Light is scattered by these particles, leading to an effective background absorbance. The in-line absorbance offset limits the dynamic range of the measurement and obscures the true absorbance value. Standard analytical sample preparation (centrifugation or membrane filtration) removes essentially all particles above a dimension of $0.45 \mu \mathrm{m}$. The $0.45 \mu \mathrm{m}$ membrane filters clog frequently while the $2.0 \mu \mathrm{m}$ filtration reduces the particle level to a tolerable level in the background absorbance.

The measurement focus of this study was to validate if the LED sensor is a suitable replacement for a UV-vis spectrometer in making these absorbance measurements. A plot comparing absorbance measured using the LED sensor and UV-vis for a single Shiraz fermentation is shown in Figure 4. A total of 28 samples were collected during fermentation, of which two were excluded from this analysis because of interference from carbon dioxide bubbles. The absorbance measured using
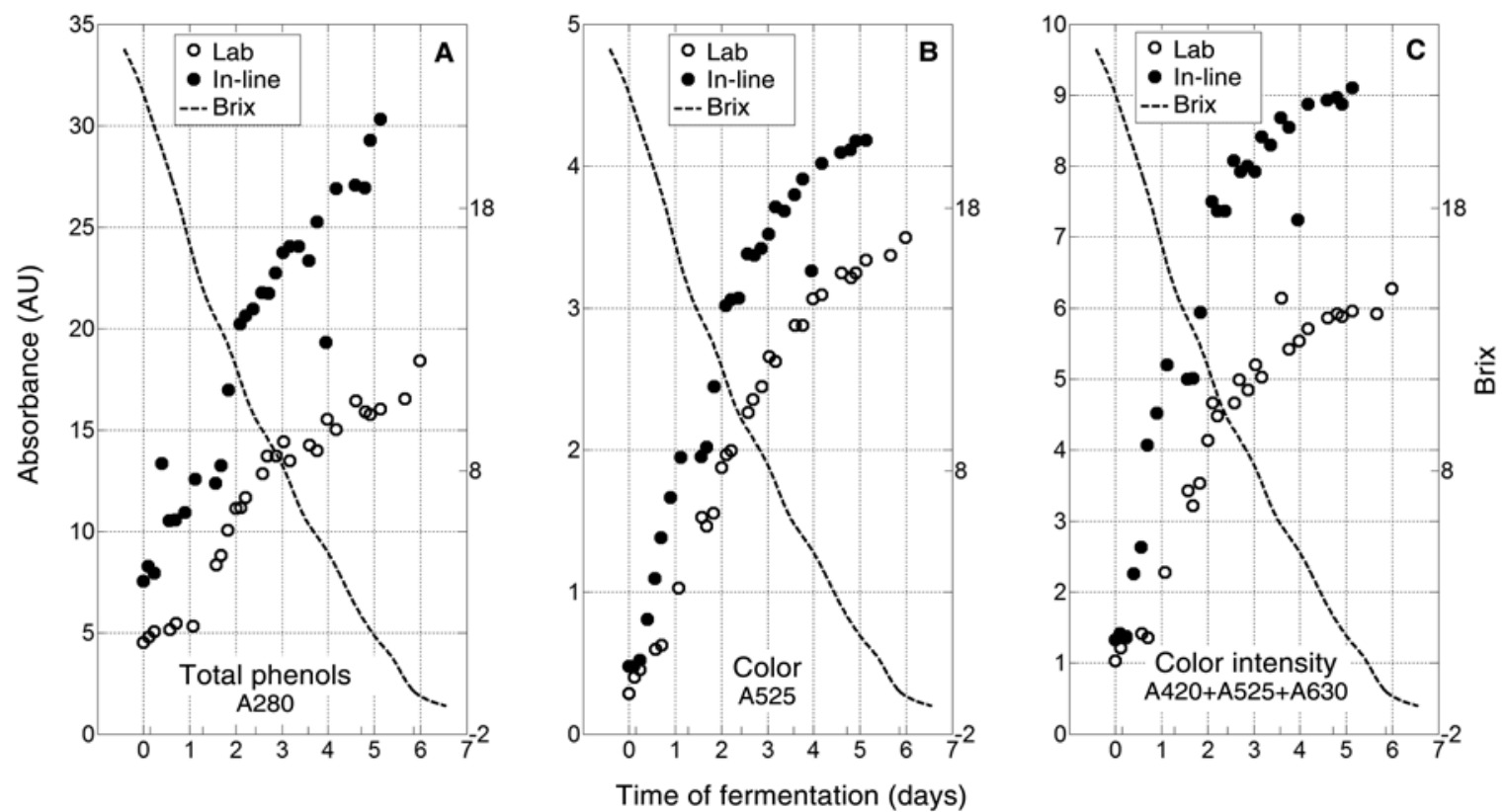

Figure 3 Extraction curves for (A) Total Phenols, (B) Color, and (C) Color Intensity for a Cabernet Sauvignon fermentation during an in-line experiment. The absorbance was measured using the LED sensor in two configurations: in-line (closed circle) and laboratory (open circle) and the Brix curve is overlaid for reference. 
the LED sensor for the wavelength $280 \mathrm{~nm}$ is smaller than the UV-vis measurement for all samples. The opposite trend was found for the $420 \mathrm{~nm}$ LED with the UV-vis measurement now smaller. The color $(525 \mathrm{~nm})$ is approximately equal to the UV-vis measurement. These results demonstrate that absorbance measurements made with LED sources are closely related to the spectral region of the measurement. The broad spectral bandwidth of LED sources results in more or less light in the measurement compared to a narrow bandwidth UVvis measurement. Several groups (Macka et al. 1996, Smith and Cantrell 2007) developed a model to relate the effect of broad bandwidth LED sources on absorbance measurements and used it to study a variety of chemical analytes. Smith and Cantrell found that the best linearity is achieved with narrow LED sources centered on broad spectral absorbance features. To test if the relationship between LED and UV-vis absorbance was systematic, a correlation study was used on all fermenta- tion samples collected during the Fall 2012 season $(n=254)$. Figure 5 shows a correlation plot comparing LED and UV-vis absorbance measurements for wavelengths 280,420 , and 525 $\mathrm{nm}$. The absorbance measurements made with LEDs showed high correlation $\left(\mathrm{R}^{2}>0.94\right)$ for both total phenols $(280 \mathrm{~nm})$ and red color $(525 \mathrm{~nm})$, but performed only adequately $\left(\mathrm{R}^{2}<\right.$ 0.75 ) for the $420 \mathrm{~nm}$ and other wavelengths. A plot showing phenolic extraction from UV-vis absorbance measurements of days 1, 3, and 6 of a red wine fermentation is shown in Figure 6. The slope and shape of the absorbance feature changes very little for the total phenols $(280 \mathrm{~nm})$ and red color $(525$ $\mathrm{nm})$ measures with regard to time of extraction. In contrast, the $420 \mathrm{~nm}$ and $630 \mathrm{~nm}$ shape of the absorbance feature changes substantially over the six fermentation days shown. The poor correlation observed is the result of making a measurement over this non-static absorbance region with a broad spectral bandwidth LED. This is consistent with previous

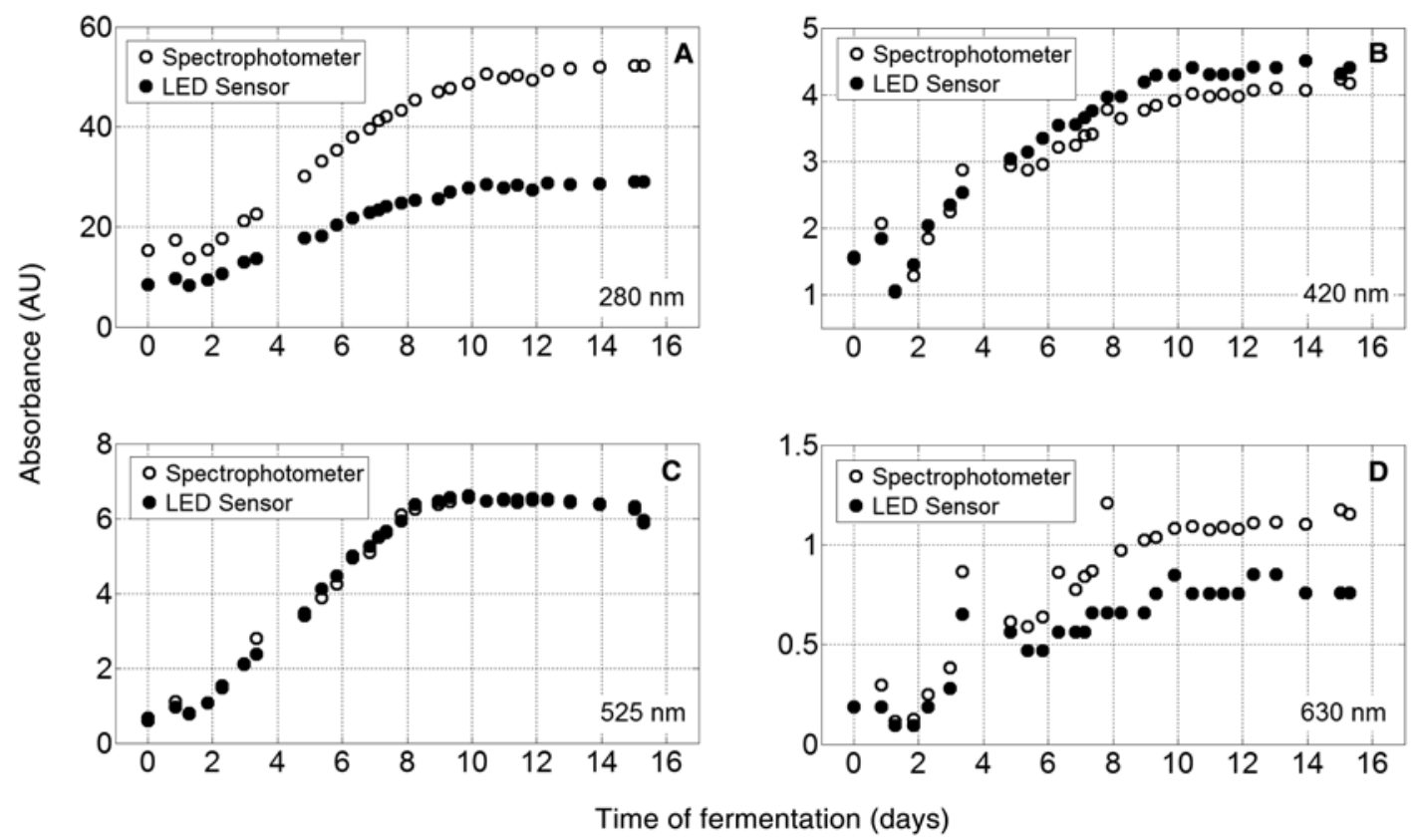

Figure 4 The absorbance measured in a Shiraz fermentation by the LED sensor (open circle) is compared against that measured by a reference UVvis spectrophotometer (closed circle). Samples were clarified using a centrifuge before analysis. Absorbance at four wavelengths: (A) $280 \mathrm{~nm}$, (B) 420 $\mathrm{nm}$, (C) $525 \mathrm{~nm}$, and (D) $630 \mathrm{~nm}$.
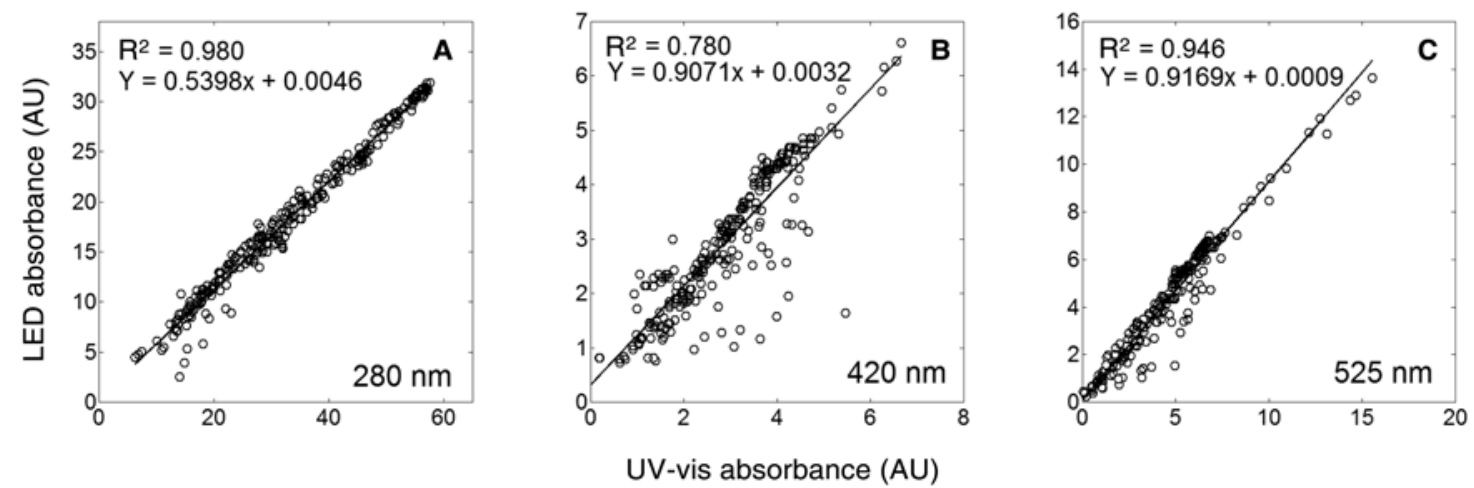

Figure 5 The absorbance measured by the LED sensor and UV-vis spectrophotometer are compared for all fermentation samples $(\mathrm{n}=256)$ : Correlation plot for wavelengths (A) $280 \mathrm{~nm}$, (B) $420 \mathrm{~nm}$, and (C) $525 \mathrm{~nm}$. 
reports (Macka et al. 1996, Smith and Cantrell, 2007) and indicates that LED absorbance measurements are dependent on the overlap of LED and absorbance spectrums. The total phenols and red color absorbance measurements made using the LED sensor were reduced by $40 \%$ and $10 \%$ respectively, compared to the UV-vis measurement. The high correlations between the two methods for the total phenols and red color allow simple corrections to be applied, translating the absorbance into an equivalent UV-vis measurement.

The phenols extracted during red wine fermentation are strongly related to the fermentation temperature. Three manually sampled fermentations of the same Shiraz fruit were fer- mented at temperatures of 15,20 , and $25^{\circ} \mathrm{C}$. The absorbance measurements for total phenols $(280 \mathrm{~nm})$ and red color $(525$ $\mathrm{nm})$ are shown in Figure 7 for a 16-day fermentation. The rate of total phenol and red color extraction is greater when the fermentation temperature is larger. The absorbance approaches a steady state saturation value for both total phenols and red color, suggesting that the rate, but not saturation value, is temperature dependent.

\section{Discussion}

The challenges encountered in developing an in-line absorbance sensor can be divided between opto-electronic and
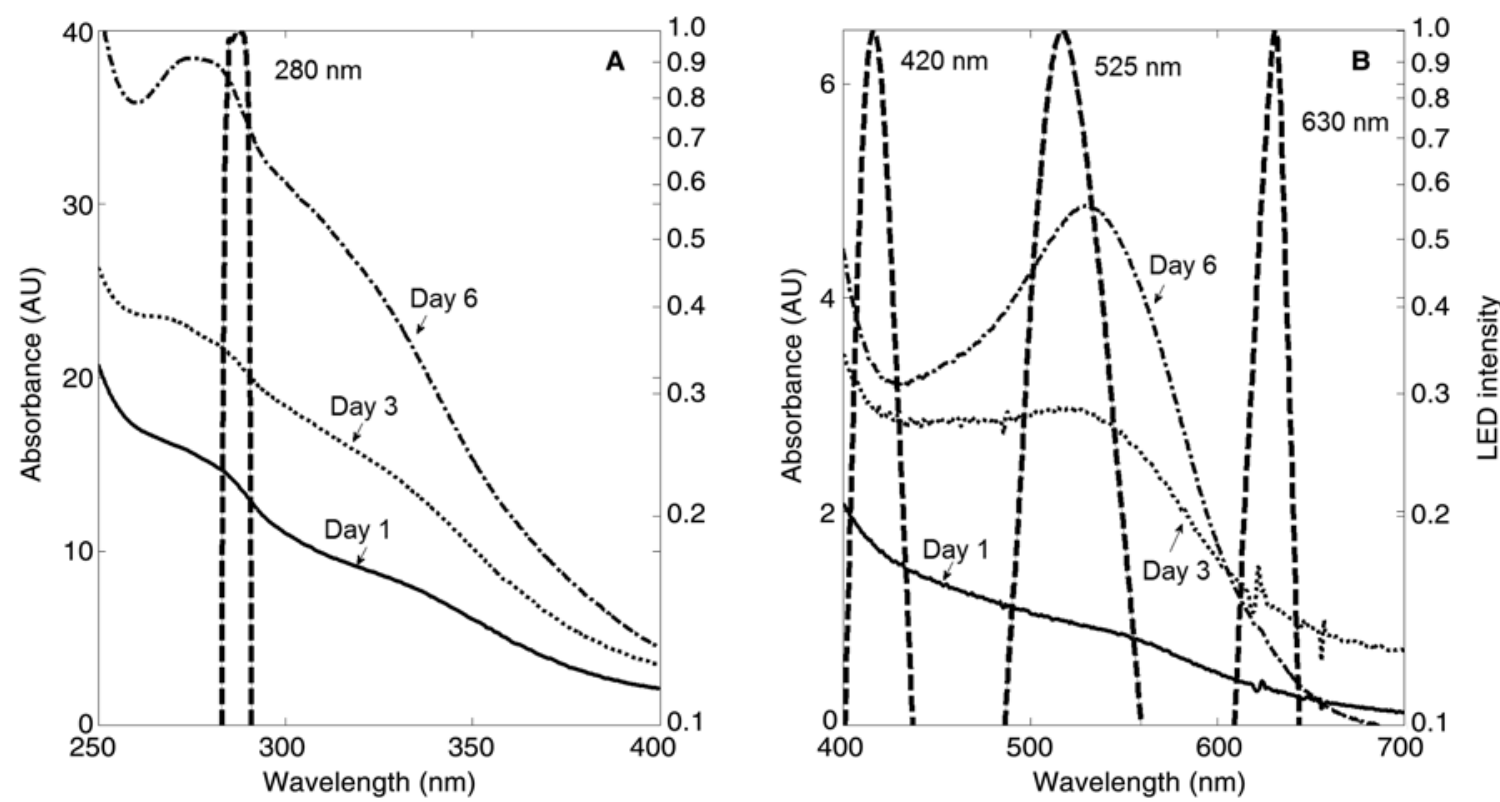

Figure 6 The power spectrum of LED light sources is measured and overlaid on some UV-vis spectra during a red wine fermentation. A represents the UV region LED and $B$ represents the three visible region LEDs.

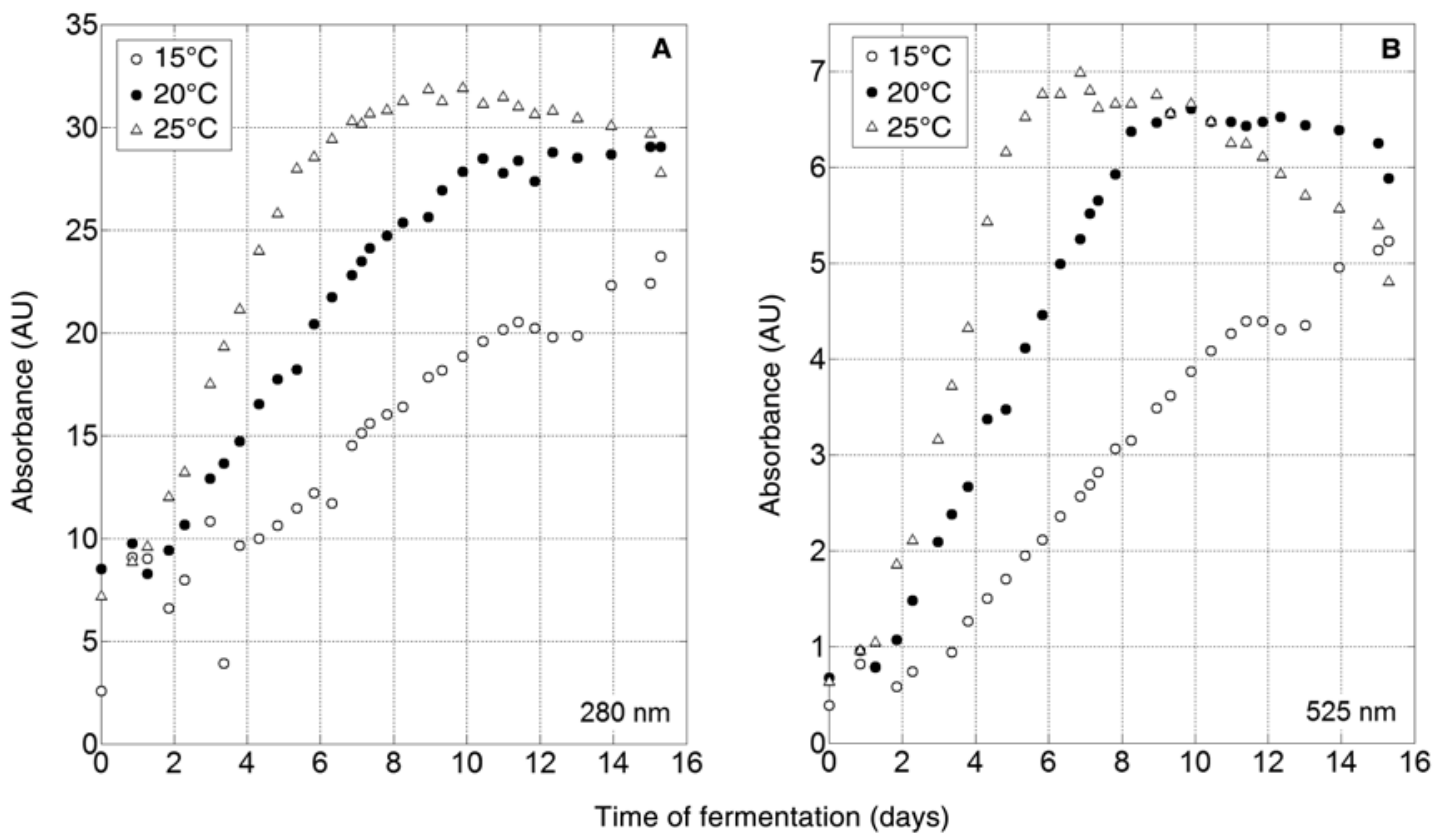

Figure 7 Extraction plot for three Shiraz fermentations with temperature treatments of 15,20 , and $25^{\circ} \mathrm{C}$ showing $(\mathbf{A})$ total phenols and $(\mathbf{B})$ red color. 
fluidic delivery issues. The opto-electronic aspect of the design required a miniaturized jig with minimal distance from LED to photodiode so as to optimize the delivery and capture of photons on the detector surface. Optimizing the photon flux onto the detector surface improves the signal to noise ratio, allowing larger optical densities to be measured. One challenge in using UV LEDs as optical sources is optimizing the light collection on the photodiode detector. The optical power of the UV LED sources is very low $(300-500 \mu \mathrm{W})$ and matters are further complicated by the poor responsitivity of the photodiodes at the UV wavelengths. The collection efficiency of the LED sensor was optimized by using large area photodiodes and minimizing the distance between LED and the detectors. The collection efficiency was good with this distance being fixed at $2.6 \mathrm{~mm}$, the thickness of the flow cell. The maximum photocurrent for the $280 \mathrm{~nm}$ LED was measured at $2 \mu \mathrm{A}$. Silicon photodiodes with UV transparent windows have a low responsivity of $0.13 \mathrm{~A} / \mathrm{W}$ at a wavelength of $280 \mathrm{~nm}$. Even under the most optimistic circumstances, in which $100 \%$ photon coupling is achieved with no reflective losses, the photocurrent would only be $65 \mu \mathrm{A}$. The existing design using a ball lens on the UV LED sources captures nearly all of the light available, as the UV LED spot size is much smaller than the UV photodiode diameter. An alternative approach is to forego the focusing ball lens and to instead use a flat window. The approach requires a large area photodiode and a very short path length between source LED and photodiode. A simple calculation shows that a flat window UV LED with 60-degree view angle and UV photodiode with $2.54 \mathrm{~mm}$ diameter would require a path length of $0.7 \mathrm{~mm}$ to capture all light. The short path length constraint adds complexity, requiring a customized optical flow cell. A ball lens is required in the current LED sensor because the thickness of the flow cell is $2.6 \mathrm{~mm}$. Future investigations might focus on miniaturized flow cells, where the ball lens is removed to reduce overall system cost.

In chemical analysis, a standard sample preparation is to filter samples using $0.45 \mu \mathrm{m}$ to remove all suspended particles and microorganisms. Using a $0.45 \mu \mathrm{m}$ filter for in-line sample clarification of must is difficult, with frequent clogging as yeast and pulp accumulate on the membrane surface. Offset correction schemes using reference wavelengths to monitor the extent of optical scattering have been adapted for in-line processes (Merzlyak et al. 2000, Paz et al. 2002). The $630 \mathrm{~nm}$ LED used in this sensor did not successfully quantify the optical scattering in these red wine fermentation measurements. As shown in Figure 4, the change in absorbance of $0.75 \mathrm{AU}$ (in a $1 \mathrm{~cm}$ path length) for the $630 \mathrm{~nm}$ LED wavelength is not in the instruments' noise, and represents a real change in the measured absorbance of roughly $1 \%$ transmittance. This $1 \%$ change in transmittance is likely attributed to the shoulder of the color absorbance. Another option would be the monitor at a reference wavelength further in the near infrared region (750 to $2500 \mathrm{~nm}$ ) for an estimate of the optical scattering. In this region, absorbance is essentially independent of phenol or color contributions. The use of background correction schemes using reference wavelength absorbance measure- ments is advocated because it might reduce the in-line filtration requirement. This is important because it will ensure filter life during each fermentation. A $2.0 \mu \mathrm{m}$ pore filter in a $47 \mathrm{~mm}$ housing was used to remove gross particulates from the sampled wine, and effectively removed the major portion of this background absorbance contribution.

The problem of carbon dioxide release leading to bubble formation in the flow cell was also addressed by the in-line filtration under pressure of the peristaltic pump. Bubbles can obscure the path length and lead to random variation in the measured absorbance. No additional approach was made in the current experimental setup to avoid or remove bubbles. One approach described for HPLC-based capillary system was sufficient backpressure to avoid outgassing and bubble formation (Wang et al. 2000). Another approach might be to adopt an open-cell dip-type reflectance probe where the sample could be agitated to remove bubbles. Bubble formation is most prevalent $\sim 12$ to $36 \mathrm{hr}$ after inoculation of the fermentation, when the Brix begins dropping most rapidly. To address bubble interference in the in-line trials, the measurements were repeated until consistent results were achieved. In repeating the measurement, the wine pump was turned on to flush the cell with a new sample. Sample measurements are judged to be of poor quality when the standard deviation for any LED wavelength is poor $(>0.36 \%$ variation from the mean). The primary concern in repeating sample measurements is that it requires frequent replacement of the filter due to a build-up of particles on the membrane surface. Reducing the sample volume or the sampling frequency is critical for prolonging the membrane filter for in-line measurements. Future studies might investigate minimizing the volume of wine that flows across the filter to ensure its lifetime during fermentation.

\section{Conclusion}

An LED sensor capable of measuring total phenols and red color in real-time during red wine fermentations is described. The sensor can make absorbance measurements at eight wavelengths: six in the visible, and two in the UV ranges. A short path-length flow cell was connected to a peristaltic pump to analyze undiluted fermentation samples. The performance of the sensor was tested in two configurations (in-line and laboratory) for multiple red wine fermentations. The high correlation found for total phenol $(280 \mathrm{~nm})$ and red color $(525 \mathrm{~nm})$ between the LED sensor and a laboratory spectrophotometer demonstrates the ability to replace a UV-vis spectrophotometer with the sensor.

\section{Literature Cited}

Beaton, A.D., V.J. Sieben, C.F.A. Floquet, E.M. Waugh, S. Abi Kaed Bey, I.R.G. Ogilvie, M.C. Mowlem, and H. Morgan. 2011. An automated microfluidic colourimetric sensor applied in situ to determine nitrite concentration. Sensors Actuators B: Chem. 156:1009-1014.

Boulton, R. 2001. The copigmentation of anthocyanins and its role in the color of red wine: A critical review. Am. J. Enol. Vitic. 52:67-87.

Brossaud, F., V. Cheynier, and A.C. Noble. 2001. Bitterness and astringency of grape and wine polyphenols. Aust. J. Grape Wine Res. 7:33-39. 
Cozzolino, D., M.J. Kwiatkowski, M. Parker, W.U. Cynkar, R.G. Dambergs, M. Gishen, and M.J. Herderich. 2004. Prediction of phenolic compounds in red wine fermentations by visible and near infrared spectroscopy. Anal. Chim. Acta 513:73-80.

Dasgupta, P.K., I.Y. Eom, K.J. Morris, and J. Li. 2003. Light emitting diode-based detectors: Absorbance, fluorescence and spectroelectrochemical measurements in a planar flow-through cell. Anal. Chim. Acta 500:337-364.

Donovan, J.L., A.S. Meyer, and A.L. Waterhouse. 1998. Phenolic composition and anti-oxidant activity of prunes and prune juice (Prunus domestica). J. Agric. Food Chem. 46:1247-1252.

Peng, Z., P.G. Iland, A. Oberholster, M.A. Sefton, and E.J. Waters. 2002. Analysis of pigmented polymers in red wine by reverse phase HPLC. Aust. J. Grape Wine Res. 8:70-75.

Harbertson, J.F., and S. Spayd. 2006. Measuring phenolics in the winery. Am. J. Enol. Vitic. 57:280-288.

Harbertson, J.F., E.A. Picciotto, and D.O. Adams. 2003. Measurement of polymeric pigments in grape berry extracts and wines using a protein precipitation assay combined with bisulfite bleaching. Am. J. Enol. Vitic. 54:301-306.

Hauser, P.C., T.W.T. Rupasinghe, C.C. Lucas, and A. McClure. 1995. Process monitor for an ammoniacal nickel solution employing an infrared light-emitting diode and a log-ratio amplifier. Analyst 120:2635-2638.
Macka, M., P. Andersson, and P.R. Haddad. 1996. Linearity evaluation in absorbance detection: The use of light-emitting diodes for on-capillary detection in capillary electrophoresis. Electrophoresis 17:1898-1905.

Merzlyak, M.N., and K.R. Naqvi. 2000. On recording the true absorption spectrum and the scattering spectrum of a turbid sample: application to cell suspensions of the cyanobacterium Anabaena variabilis. J. Photochem. Photobiol., B 58:123-129.

Paz, I., and G. Pinto. 2002. Spectroscopic study about the kinetics of the anthocyanin pigments extraction during the maceration of cherries in liquor. Spectrosc. Lett. 35:357-368.

Skogerson, K., M. Downey, M. Mazza, and R. Boulton. 2007. Rapid determination of phenolic components in red wines from UV-visible spectra and the method of partial least squares. Am. J. Enol. Vitic. 58:318-325.

Smith, R., and K. Cantrell. 2007. Modeling the effect of polychromatic light in quantitative absorbance spectroscopy. J. Chem. Educ. 84:1021.

Somers, T.C. 1998. The Wine Spectrum. Hyde Park Press, Adelaide.

Thorngate, J.H., and A.C. Noble. 1995. Sensory evaluation of bitterness and astringency of $3 \mathrm{R}(-)$-epicatechin and $3 \mathrm{~S}(+)$-catechin. J. Sci. Food Agric. 67:531-535.

Wang, H., C.Y. Eugene, C.A. Ibarra, and M. Hackett. 2000. A remote flow cell for UV absorbance detection with capillary HPLC based on a single strand fiber optic. Analyst 125:1061-1064. 